\title{
No toda hernia lumbar causa lumbalgia
}

Delia Gil Huayanay ${ }^{1, a}$, Vicente Benites Zapata ${ }^{2, b}$, Carlos Alberto Ramirez La Torre ${ }^{3, b}$

\section{RESUMEN}

Tras una larga serie de términos empleados para definir el desplazamiento del material discal (ya sea del núcleo pulposo o del anillo fibroso), se clasificó a las hernias discales en abombamiento, protrusión focal, extrusión y secuestro. La hernia discal representa entre el $1 \%$ al $3 \%$ de todas las causas de lumbalgia. Sin embargo, no todas las hernias discales son necesariamente sintomáticas: la presencia o ausencia de sintomatología depende del tamaño, localización y extensión del material discal herniado en relación con el conducto raquídeo, y de si compromete o no las raíces nerviosas. Durante la década de los 80 y 90 se realizaron numerosos estudios de resonancias magnéticas y tomografías a personas asintomáticas, descubriéndose que un número significativo de estas personas que no padecían ningún tipo de dolor tenían protrusiones y abombamiento de discos.

Palabras clave: Hernia discal; lumbalgia; desplazamiento del disco intervertebral (Fuente: DeCS BIREME).

\section{Not all lumbar herniated discs cause low back pain}

\section{ABSTRACT}

After a long series of terms used to define the displacement of disc material (either the nucleus pulposus or the fibrous ring), herniated discs were classified as bulging, focal protrusion, extrusion and sequestration. Herniated discs represent from $1 \%$ to $3 \%$ of all causes of low back pain. However, not all herniated discs are necessarily symptomatic: the presence or absence of symptoms depend on the size, location and extent of the herniated disc material in relation to the spinal canal, and whether or not it compromises the nerve roots. Several studies using magnetic resonance imaging and CT scans were performed during the 1980s and 1990s to asymptomatic individuals, and a significant number of these individuals who did not have any type of pain had disc protrusion and bulging.

Keywords: Heniated disc; low back pain; intervertebral disc displacement (Source: MeSH NLM).

1. Médico Ocupacional, egresada de la Maestría de Salud Ocupacional y Ambiental.

2. Maestro en Investigación Epidemiológica, Delegado Académico de la Maestría de Investigación Clínica.

3. Médico Ocupacional, docente de la Maestría de Medicina Ocupacional y Medio Ambiente.

a. Universidad Nacional Mayor de San Marcos.

b. Facultad de Medicina Humana, Universidad de San Martín de Porres. 


\section{INTRODUCCIÓN}

Se realizó una búsqueda bibliográfica utilizando los siguientes encabezamientos MeSH: Desplazamiento de disco intervertebral, lumbalgia y hernia discal. En base a los artículos encontrados es que se elaboró el presente artículo de revisión, cuyos resultados se exponen a continuación.

La terminología para las alteraciones del disco intervertebral es variada y amplia, lo cual genera confusión e incoherencias entre los distintos especialistas médico-quirúrgicos ${ }^{(1-3)}$.

En el año 2001, la Sociedad Norteamericana de Columna, la Sociedad Americana de Radiología de Columna y la Sociedad Americana de Neurorradiología, impulsaron la creación de un léxico estandarizado para describir la patología discal lumbar. Esta iniciativa fue aprobada por la Sociedad Americana de Neurocirujanos, el Congreso de Neurocirujanos, y el Comité de Codificación Internacional de la Academia Americana de Traumatólogos convirtiéndose en la más recomendada hoy en día para la descripción de este tipo de patología. La clasificación de las lesiones discales es la siguiente: normal, variante normal, anomalía del desarrollo o congénita, degenerativa / traumática, inflamatoria / infecciosa y neoplásica (4-5). En el año 2014, publicaron la última versión de la nomenclatura para describir la patología discal lumbar ${ }^{(6)}$.

Las lesiones degenerativas / traumáticas pueden ser fisiológicas (envejecimiento discal, con afectación difusa de todos los discos, sobre todo los lumbares inferiores por causas mecánicas) o no (degeneración discal), que afecta como máximo a tres discos y está producida por una desecación acelerada, atrofia y transformación fibrosa del disco con importante alteración del platillo terminal ${ }^{(7)}$.

El disco es una combinación de tejidos conectivos fuertes que unen una vértebra a la siguiente y actúa como un cojín entre las vértebras. El disco está hecho de una capa externa dura conocida como anillo fibroso, y un gel central conocido como núcleo pulposo. A medida que envejece, su centro puede comenzar a perder el contenido de agua, haciendo menos eficaz su función de cojín. Esto puede causar un desplazamiento del centro del disco (hernia), a través de una grieta en la capa exterior ${ }^{(8,9)}$.

Tras una larga serie de términos empleados para definir el desplazamiento del material discal, se clasificó a las hernias discales en abombamiento, protrusión focal, extrusión y secuestro. La diferencia radica en la cantidad de disco desplazado ${ }^{(10)}$.

La hernia discal representa entre el 1 al $3 \%$ de todas las causas de lumbalgia ${ }^{(8)}$. No todas las hernias discales son necesariamente sintomáticas, la presencia o ausencia de sintomatología depende del tamaño, localización y extensión del material discal herniado en relación con el conducto raquídeo y su compromiso o no de las raíces nerviosas ${ }^{(11)}$.
Durante la década de los 80 y 90 se realizaron numerosos estudios de resonancias magnéticas (RMN) y tomografías a personas asintomáticas, descubriéndose que un número significativo de estas personas que no padecían ningún tipo de dolor, tenían protrusiones y abombamiento de discos ${ }^{(12-19)}$.

Aquella hipótesis de que las personas asintomáticas con presencia de hernia del disco, evidenciada en las resonancias magnéticas, podrían desarrollar dolor lumbar y/o ciatalgia, fue derribado en el año 2001 por Borenstein, quien denominó a todos estos casos como hernia falsos positivos. A cada uno de estos casos se les hizo seguimiento durante siete años para ver si al cabo de este tiempo se convertirían en casos sintomáticos. Al finalizar dicho periodo se dieron con la sorpresa que ninguno de los casos se volvió sintomático ${ }^{(18)}$.

\section{Estudios de investigación relevantes}

En 1976, Paajanen realizó resonancias magnéticas a un grupo de adultos jóvenes, encontrando que el $57 \%$ de los pacientes con lumbalgia tenían uno o más discos con degeneración y un 35\% de los pacientes asintomáticos presentaban degeneración discal significativa ${ }^{(19)}$.

Otra investigación muy comentada es la de Miller, quien correlacionó los grados macroscópicos de degeneración discal con edad, sexo y columna vertebral en 600 discos intervertebrales, encontrándose que los discos del sexo masculino estaban más degenerados significativamente en aquellos mayores de 50 años ${ }^{(20)}$.

La presencia de degeneración discal y la presencia o ausencia de dolor lumbar puede ser considerada análoga a la aparición de cálculos biliares y dolor abdominal. Aunque la mayoría de los individuos con cálculos biliares son asintomáticos, si un paciente tiene cálculos biliares y presenta los síntomas característicos, éstos pueden atribuirse a los cálculos biliares ${ }^{(21)}$.

En el año 1995, 56 pacientes con hernia de disco lumbar participaron de un estudio doble ciego en donde se quiso comparar los resultados de la inyección de quimopapaína versus la solución salina para el tratamiento de la hernia discal. Los participantes fueron invitados a someterse a una evaluación por resonancia magnética de columna lumbosacra 10 años después. Los resultados indicaron que la mejora a largo plazo de los síntomas de un paciente después del tratamiento de la hernia discal puede ocurrir con o sin resolución de la hernia, es decir, que aquellos que afirmaban sentirse bien aún mantenían la hernia del disco. ${ }^{(22)}$.

En el año 2005, Masui y colaboradores publicaron un estudio de investigación sobre el resultado clínico de 27 pacientes que fueron tratados de forma conservadora (sin cirugías) para la hernia de disco lumbar sintomática. Los estudios de seguimiento por resonancia magnética se completaron a los 2 y 7 años. A los 7 años de seguimiento, se observó degeneración progresiva del disco en todos los 
pacientes; sin embargo, no hubo correlación predictiva entre los hallazgos de la resonancia magnética y la persistencia del dolor. Los autores concluyeron que el resultado clínico no dependió del tamaño de la herniación o del grado de degeneración del disco intervertebral en el seguimiento mínimo de 7 años ${ }^{(23)}$.

Uno de los estudios muy citados fue el de Boden, quien realizó resonancias magnéticas a 67 pacientes que nunca habían padecido de dolor lumbar. Se encontró que el $20 \%$ de estas personas asintomáticas con una edad menor a 60 años tenían hernia discal, la misma que se confirmaba mediante el resultado de resonancia magnética. En el grupo que tenía edades mayores a 60 años, se encontró que el 37\% tenía hernias de disco confirmadas y el $21 \%$ tenía estenosis espinal a pesar de no tener dolor. Desafortunadamente, estos investigadores no diferenciaron entre una protrusión (herniación contenida) y una extrusión (herniación no contenida). Otro punto de interés, fue el hecho de que el $35 \%$ de estas personas asintomáticas, entre las edades de 20 y 39 años, tenían enfermedad degenerativa del disco con o sin protuberancia de disco ${ }^{(13)}$.

En 1994, Jensen y colaboradores encontraron que el $52 \%$ de los 98 pacientes asintomáticos estudiados, presentaron una protuberancia de disco en los resultados de resonancia magnética y el $27 \%$ presentó una protrusión del disco. Sólo el $1 \%$ de los pacientes tenían una extrusión de disco; demostrándose así que la resonancia magnética es bastante precisa para detectar las extrusiones de disco. De los 98 voluntarios estudiados, el 64\% tenía un disco anormal en los resultados de resonancia magnética (abombamiento, protrusión y / o extrusión). También de interés, fueron los siguientes hallazgos en estos sujetos asintomáticos: ruptura de las fibras externas del anulus, 14\%; enfermedad degenerativa de las articulaciones facetarias, 8\%; espondilolisis, 7\%, espondilolistesis, $7 \%$; y estenosis central $7 \%{ }^{(12)}$.

En el año 1998, Weishaupt y Boos seleccionaron al azar a un grupo de 60 personas que nunca habían padecido anteriormente lumbalgia. Entre los seleccionados estaban algunos trabajadores de un centro hospitalario y pacientes que no tenían patología lumbar. Veinte de los seleccionados tenían edades entre 20 a 29 años, otros veinte tenían edades entre 30 a 39 años, y los últimos veinte tenían entre 40 y 49 años; siendo éste un grupo muy joven ya que la edad media del estudio fue de 35 años. Cada paciente fue sometido a una resonancia magnética y los resultados fueron interpretados por dos radiólogos diferentes.

Finalmente, se encontró que el $24 \%$ tenía abombamiento del disco; el $40 \%$ presentaba protrusión del disco y el $18 \%$ tenía extrusión del disco, que es una forma severa de la herniación del disco. Ninguno de los participantes presentó secuestro del disco. Con respecto a la compresión de las raíces nerviosas, el 23\% tenía contacto con las raíces nerviosas sin desplazamiento, el 5\% tenía desplazamiento real de la raíz nerviosa (hernia discal) sin compresión física severa y el $2 \%$ demostró una compresión completa de las raíces nerviosas ${ }^{(14)}$.
En el año 1995, Boos trabajó con voluntarios asintomáticos de alto riesgo y falsos positivos en las imágenes de resonancias magnéticas. Para esta investigación tomó a un grupo de 46 personas asintomáticas que realizaban actividades de alto riesgo y escanearon su columna lumbar con resonancia magnética. Todos estos voluntarios realizaban actividades de alto riesgo (flexiones frecuentes, torsión frecuente, manipulación frecuente de carga, exposición frecuente a vibraciones) para el desarrollo de hernia discal sintomática.

Los resultados indicaron que el $76 \%$ de los estudiados tenían al menos una alteración discal (protrusión o extrusión); sin embargo, cabe destacar el hecho de que sólo el $13 \%$ de estas hernias fueron del tipo más severo, la extrusión. No se encontró secuestro de disco en el grupo asintomático.

Con respecto a la compresión de la raíz nerviosa por la hernia discal, el $17 \%$ de las personas asintomáticas tenía un menor compromiso (contacto o desviación de la raíz), mientras que sólo el $4 \%$ tenía compromiso mayor (compresión de la raíz).

Con respecto a la enfermedad degenerativa del disco, el 85\% del grupo asintomático había confirmado la degeneración discal en la resonancia magnética en al menos un nivel, es decir, grado 3, 4 ó 5, según lo definido por Pearce ${ }^{(15)}$.

Este estudio demuestra que la hernia del disco por sí sola no equivale a lumbalgia y/o ciatalgia. El $76 \%$ de estas personas asintomáticas de alto riesgo demostraron protrusión del disco o incluso extrusión (13\%) en la resonancia magnética, sin embargo no tenían lumbalgia o ciatalgia. Es evidente que existen otros factores que contribuyen al fenómeno de la lumbalgia.

En el 2005, Quiroz analizó 105 casos de resonancia magnética de columna lumbar de individuos asintomáticos. Todos los estudios fueron realizados con un mismo resonador e interpretados por un médico radiólogo experimentado quien no tenía conocimiento de la condición clínica de los pacientes. Para no influir en los resultados se mezclaron casos de sujetos con lumbalgia baja, de tal manera que en cada sesión de interpretación se analizaron aproximadamente 16 estudios, de los cuales la mitad correspondía a sujetos sanos y el resto a pacientes con sintomatología; sin embargo, estos últimos no se incluyeron dentro del análisis estadístico. De los 105 casos estudiados, el $45 \%$ fueron normales y el 55\% presentaron alteraciones discales. Del total de casos asintomáticos, en 40 participantes (38\%) se observó abombamiento discal en al menos un nivel y en 18 (17\%) se observó protrusión. En ninguno se observó, extrusión discal ${ }^{(24)}$.

La tabla 1 muestra los porcentajes de alteraciones discales en las lecturas de resonancia magnética lumbar, según cada categoría (abombamiento, protrusión o extrusión). Hay que recordar que ninguno de las personas estudiadas había tenido lumbalgia significativa y/o ciatalgia. 
Tabla 1. Porcentajes de alteraciones discales en RMN lumbar de pacientes asintomáticos

\begin{tabular}{|c|c|c|c|c|}
\hline Investigación & Abombamiento & Protrusión & Extrusión & $\begin{array}{l}\text { Desviación o } \\
\text { compresión de raíz }\end{array}$ \\
\hline Boden & & $20 \%$ & & \\
\hline Jensen & $52 \%$ & $27 \%$ & $1 \%$ & \\
\hline Quiroz & $38 \%$ & $17 \%$ & & \\
\hline Weishaupt & $24 \%$ & $40 \%$ & $18 \%$ & $4 \%$ \\
\hline Promedios & $37,5 \%$ & $25,7 \%$ & $9,2 \%$ & $4 \%$ \\
\hline
\end{tabular}

En conclusión, existen personas asintomáticas con presencia de alteraciones de la columna lumbar. Según los estudios revisados, hasta un $37,5 \%$ de personas asintomáticas presentaron abombamiento del disco, un $25,7 \%$ protrusiones y un $9,2 \%$ extrusiones. En base a estos estudios de investigación realizados en las últimas décadas se presume que las alteraciones discales, no necesariamente, son la causa de las lumbalgias. Debería pensarse en la existencia de otros factores que estén contribuyendo a la aparición de la lumbalgia.

Según el estudio de Boos, se evidenció mediante resonancia magnética que el $76 \%$ de los 46 trabajadores asintomáticos estudiados y que realizaban actividades de alto riesgo tales como flexiones frecuentes, torsión frecuente, manipulación frecuente de carga y exposición frecuente a vibraciones, presentaron al menos una alteración discal sin sintomatología.

El estudio con mayor número de población asintomática fue el de Quiroz realizado en el año 2005 donde se evaluó mediante la resonancia magnética a 105 colaboradores, encontrándose que el $55 \%$ presentaba alteraciones discales.

Con el fin de incrementar la potencia estadística de los estudios en personas asintomáticas es recomendable replicar las mismas investigaciones considerando un mayor número de tamaño muestral.

\section{REFERENCIAS BIBLIOGRÁFICAS}

1. Fardon D, Balderston R, Garfin S, Nasca R, Pinkerton S, Salib R. Disorders of the Spine, a coding system for diagnoses. Philadelphia: Hanley and Belfus; 1991.

2. Fardon D, Balderston R, Garfin S, Nasca R, Pinkerton S, Salib R. Terms used for diagnosis by English speaking spine surgeons. Spine. 1993;18(2):274-7.

3. American Academy of Orthopaedic Surgeons. A glossary on spinal terminology. Chicago: The Academy; 1985:31-32.

4. Swartz J. Letter from the guest editor: protrusion, extrusion... confusion! Seminars in Ultrasound, CT and MRI. 1993;14(6):383-4.

5. Boo S, Hogg J. How's your disk? Illustrative glossary of degenerative disk lesions using standardized lexicon. Curr Probl Diagn Radiol, 2010;39(3):118-24.

6. Fardon D, Williams A, Dohring E, Murtagh R, Rothman G, Sze G. Recommendations of the combined task forces of the North American Spine Society, the American Society of Spine Radiology and the American Society of Neuroradiology. Spine

\section{J. 2014;14(11):2525-45.}

7. Milette P, Melançon D, Dupuis P, Vadeboncoeur R, Bertrand G, Pelletier J, et al. A simplified terminology for abnormalities of the lumbar disks. J Radiol. 1992;73(12):645-51.

8. Beard H, Stevens R. Biochemical changes in the intervertebral disc. En: Jayson MV, editors. The Spine and Backache. London: Pitman;1980. p. 407-36.

9. Bogduk N. The interbody joint and the intervertebral disc. En: Bogduk N, editor. Clinical anatomy of the lumbar spine and sacrum. London: Elsevier;2008. p. 11-28.

10. Paajanen H, Erkintalo M, Kuusela T, Dahlstrom S, Kormano M. Magnetic resonance study of disc degeneration in young lowback pain patients. Spine. 1989:14(9):982-5.

11. Ackerman J, Steinberg P, Bryan N, BenDebba M, Long D. Persistent low back pain in patients suspected of having herniated nucleus pulposus: radiologic predictors of functional outcome implications for treatment selection. Radiology 1997;203(3):815-22.

12. Jensen M, Brant-Zawadzki M, Obuchowski N, Modic M, Malkasian D, Ross J. Magnetic resonance imaging of the lumbar spine in people without back pain. N Engl J Med. 1994;331(2):369-73.

13. Boden S, Davis D, Dina T, Patronas N, Wiesel S. Abnormal magnetic resonance scans of the lumbar spine in asymptomatic subjects: A prospective investigation. J Bone Joint Surg Am.1990;72(3):403-8.

14. Weishaupt D, Zanetti M, Holdler J, Boos N. MRI of the lumbar spine: Prevalence of intervertebral disc extrusion and sequestration, nerve root compression and plate abnormalities, and osteoarthritis of the fact joints in Asymptomatic Volunteers. Radiology. 1998;209(3):661-6.

15. Boos N, Rieder R, Schade V, Spratt KF, Semmer N, Aebi M. 1995 Volvo Award in clinical science: The diagnostic accuracy of MRI, work perception, and psychosocial factors in identifying symptomatic disc herniations. Spine. 1995;20(24):2613-25.

16. Powell MC, Wilson M, Szypryt P, Symonds EM, Worthington BS. Prevalence of lumbar disc degeneration observed by magnetic resonance in symptomless women. Lancet. 1986; 328(8520):1366-7

17. Boos N, Semmer N, Elfering A, Schade V, Gal I, Zanetti M, et al. Natural history of individuals with asymptomatic disc abnormalities in MRI: Predictors of low back pain-related medical consultation and work incapacity. Spine. 2000; 25(12):1484-92.

18. Borenstein G, O'Mara W, Boden D, Lauerman C, Jacobson A, Platenberg $C$, et al. The value of magnetic resonance imaging of the lumbar spine to predict low-back pain in asymptomatic individuals: A 7-year follow-up study. J Bone Joint Am. 2001;83(9):1306-1.

19. Wiesel SW, Tsourmas N, Feffer HL, Citrin CM, Patronas N. A study of computer-associated tomography: I. The incidence of positive CAT scans in asymptomatic group of patients. Spine. 1984;9(6):549-51. 
20. Miller A, Schmatz C, Schultz B. Lumbar disc degeneration: correlation with age, sex, and spine level in 600 autopsy specimens. Spine. $1998 ; 13(2): 173-8$.

21. Baldwin, N. Lumbar disc disease: the natural history. Neurosurg. Focus. 2002;13(2):E2.

22. Fraser D, Sandhu A, Gogan J. Magnetic resonance imaging findings 10 years after treatment for lumbar disc herniation. Spine. 1995;20(6):710-4.

23. Masui T, Yukawa Y, Nakamura S, Kajino G, Matsubara Y, Kato F, et al. Natural History of Patients with Lumbar Disc Herniation Observed by Magnetic Resonance Imaging for Minimum 7 Years. J Spinal Disord Tech. 2005;18(2):121-6.

24. Quiroz R, Lezama G, Gómez C. Alteraciones discales de columna lumbar identificadas por resonancia magnética en trabajadores asintomáticos. Rev Med Inst Mex Seguro Soc. 2008;46(2):185-90.
Fuentes de financiamiento:

Este artículo ha sido financiado por los autores.

\section{Conflictos de interés:}

Los autores declaran no tener ningún conflicto de interés.

\section{Correspondencia:}

Delia Gil Huayanay.

Dirección: Jr. Pumacahua 1616-2016 Jesús María (Lima Perú). Teléfono: +51992193833

Correo electrónico: delia.gil.h@gmail.com

Recibido: 02 de mayo de 2017
Evaluado: 02 de mayo de 2017
Aprobado: 31 de mayo de 2017

(c) La revista. Publicado por Universidad de San Martín de Porres, Perú. (cc) Br Licencia de Creative Commons Artículo en acceso abierto bajo términos de Licencia Creative Commons Atribución 4.0 Internacional. (http://creativecommons.org/licenses/by/4.0/) 